\title{
Von der Vetokammer zum deliberativen Organ? Die ersten Senatswahlen in Thailand ${ }^{1}$
}

\author{
Von Aurel Croissant
}

\section{Einleitung}

Am 4. März und 29. April diesen Jahres fanden in Thailand die beiden ersten Runden der ersten Senatswahl seit Einführung des Zweikammerparlamentes im Jahre 1946 statt. Im Vorfeld wurden die ersten nationalen Wahlen nach Inkrafttreten der 16. Verfassung des Königreichs im Oktober 1997 allgemein als Testfall für die Umsetzung der neuen Verfassung angesehen. Zahlreiche an der Verfassungsgebung beteiligte Personen, zivilgesellschaftliche Gruppen, die thailändischen Medien sowie ausländische Beobachter verknüpften mit der Wahl die Hoffnung, die traditionell der politischen Einflußnahme von Bürokratie und Militär dienende zweite Kammer werde aus der Wahl als überparteiliches und kompetentes Kontroll- und Beratungsorgan gegenüber dem Repräsentantenhaus und der Regierung hervorgehen. Im Zentrum der folgenden Analyse steht daher die Frage, ob die Wahl diese Ansprüche erfüllt hat und welche Rückschlüsse sich hieraus für die zukünftige politische Rolle des Senats ergeben. Zu ihrer Beantwortung wird in einem ersten Schritt die Stellung des Senats im politischen Systems Thailands vor 1997 und nach der Verfassungsreform untersucht. In einem zweiten Schritt werden die organisatorischen Rahmenbedingungen der Wahl dargestellt, um in einem dritten Schritt Ablauf und Ergebnis der Wahlen $\mathrm{zu}$ analysieren. Den Abschluß bilden einige allgemeine Überlegungen zur Bedeutung dieser Wahl für die weitere Entwicklung der thailändischen Demokratie.

\section{Die Stellung des Senats vor und nach 1997}

Die heutige thailändische Demokratie ist das Ergebnis eines durch häufige Brüche und autokratische Regressionen geprägten, langgestreckten Prozesses der Systemtransformation von der absoluten Monarchie zur konstitutionell-parlamentarischen Demokratie. Die Herrschaftsträger der autoritären Regime, die seit den 30er Jahren fast ununterbrochen die Hilfe bei der Beschaffung notwendiger Informationen sowie Uwe Solinger für seine hilfreichen und kritischen Anregungen in mehreren Gesprächen mit dem Autor 
politischen Geschicke des Landes bestimmten, waren die zivile Bürokratie und das thailändische Militär. Obwohl sich immer wieder "semi-demokratische" Phasen mit autokratischen Herrschaftsperioden abwechselten, befand sich das politische Machtzentrum des Landes stets in der zivilen und militärischen Bürokratie. Die durch ökonomischen und sozialen Wandel bedingte Schwächung staatlicher Akteure - am deutlichsten hervortretend in den politischen Erschütterungen der 70er Jahre ${ }^{2}$ - führten jedoch seit den späten 70er Jahren zu einer vorsichtigen Öffnung der "bureaucratic polity" ${ }^{3}$. Es kam zur Institutionalisierung eines "weichen" autoritären Systems, das in der Literatur als "demi-" oder "semi-democracy" ${ }^{4}$ bezeichnet wird: Eine starke Exekutive mit einem nicht gewählten Premier an der Spitze, zumeist ein ehemaliger Militär, kontrollierte den politischen Entscheidungsprozeß; das aus freien Wahlen hervorgegangene Repräsentantenhaus mußte sich seine Kompetenzen mit einem nicht demokratisch legitimierten Senat teilen, dessen Mitglieder mehrheitlich der zivilen Bürokraten und dem Militär entstammten.

\section{$1.1 \quad$ Der Senat vor 1997}

Innerhalb der formal-institutionellen Architektur wechselnder autoritärer Regime kam dem Senat eine besondere Bedeutung zu. Die Ernennung der Senatsmitglieder diente den bürokratisch-militärischen Eliten als eines von mehreren Herrschaftsinstrumenten, um ihre politische Einflußnahme auch institutionell abzusichern. Gestützt auf umfangreiche politische Vorrechte und eine, ungeachtet der Fragmentierung von Militär und Bürokratie entlang funktionaler Trennlinien ${ }^{5}$ relative Interessenhomogenität seiner Mitglieder bildete der thailändische Senat eine Institution, die in erster Linie dem Schutz der Interessen der herrschenden sozialen Gruppen diente. Da weder das gewählte Repräsentantenhaus, die (gewählten) Regierungen noch die thailändischen Wähler Einfluß auf die Zusammensetzung des Senats nehmen konnten, entzogen Militär und zivile Bürokratie über den Senat

Vgl. Wyatt, David K., Thailand: A Short History, Chiang Mai 1984, S. 299-307.

Riggs, Fred W., Thailand: The Modernization of a Bureaucratic Polity, Honolulu 1966; vgl. Hewiston, Kevin, Introduction: Power, Oppositions and Democratization, in: Hewison, Kevin (ed.), Political Change in Thailand: Democracy and Participation, London / New York, 1997, S. 1-21: 7 f.; Christensen, Scott R. / Ammar Siamwalla / Pakorn Vichayanond, Institutional and Political Bases of Growth-Inducing Policies in Thailand, in: TDRI (ed.), Thailand's Boom and Bust, Bangkok, 1997, S. 21-53.

Likhit, Dhiravegin, Demi-Democracy: The Evolution of the Thai Political System, Singapore 1992; Chai-Anan, Samudavanija, A Stable Semi-Democracy, in: Diamond, Larry / Linz, Juan J. / Lipset, Seymour Martin (eds.), Politics in Developing Countries: Comparing Experiences with Democracy, 2nd ed., Boulder, 1995, S. 322-367.

So unter anderem zwischen den Teilstreitkräften Armee, Marine und Luftwaffe sowie zwischen Polizei und Streitkräften. 
zentrale politische Entscheidungsmaterien dem Zugriff der demokratisch gewählten Parlamentarier. Die folgende Übersicht (Tabelle 1) zeigt, daß Militär und Bürokratie zudem auch in der Regierung sowie dem Repräsentantenhaus zeitweise stark vertreten waren:

\section{Tabelle 1: Durchschnittlicher Anteil ziviler Bürokraten und Militärs an Regierungsposten und Parlamentsmandaten (1932-1992)}

\begin{tabular}{|c|c|c|c|c|c|c|}
\hline & \multicolumn{2}{|c|}{$\begin{array}{l}\text { Zusammensetzung } \\
\text { der Kabinette }^{1}\end{array}$} & \multicolumn{2}{|c|}{$\begin{array}{l}\text { Zusammensetzung } \\
\text { der 2. Kammer }\end{array}$} & \multicolumn{2}{|c|}{$\begin{array}{l}\text { Zusammensetzung } \\
\text { der } 1 . \text { Kammer }^{3}\end{array}$} \\
\hline & $1932-80$ & $1980-92$ & $1946-80$ & $1980-92$ & $1932-80$ & $1980-92$ \\
\hline Militärs & 36,4 & 19,9 & 30,7 & 63,1 & - & - \\
\hline Zivile Bürokraten & 42 & 14,4 & 30,7 & 22,5 & - & - \\
\hline Insgesamt & 78,4 & 34,3 & 61,4 & 85,6 & 31,2 & 19,6 \\
\hline
\end{tabular}

1 Bis März 1992; für zivile Bürokraten nur bis 1990; Angaben für Militärs ohne die Regierung Chatichai 2 (1990-91).

2 Angaben für den 1.-13. bzw. 14.-15. Senat; Angaben für zivile Bürokraten 1980-1992 beziehen sich nur auf den 15. Senat (1985-1991);.

3 Keine Angaben, ob zivile oder militärische Bürokraten oder beides; Angaben 1932-1979; 19791988; alle Angaben in \%.

Quelle: Nach Angaben in Pisan 1988; Kreuzer, Peter, Generäle in der Politik - Politische Kultur, Streitkräftekultur und das Verhalten militärischer Eliten im politischen Raum: China - Japan - Thailand, Frankfurt am Main 1995; Pasuk, Phonpaichit / Baker, Chris, Thailand's Boom and Bust, Chiang Mai, 1998; ergänzt durch eigene Berechnungen.

Die unter General Prem (Premierminister von 1980-1988) eingeleitete Liberalisierung mündete mit den Parlamentswahlen von 1988 und der Bildung einer vom Vertrauen des Parlaments abhängigen Regierung zunächst in eine kurze formaldemokratische Phase. Sie wurde aber schon im Frühjahr 1991 erneut durch den Putsch unzufriedener Militärs um General Suchinda beendet. Auf den Sturz dieses kurzlebigen autoritären Regimes im Mai 1992 folgte wie bereits nach dem Putsch im Februar 1991 eine Interimsregierung unter dem Karrierediplomaten Anand. Im Zuge der Demokratisierung wurde keine neue Verfassung ausgearbeitet, sondern die 1991 auf Betreiben der regierenden Militärjunta verkündete Verfassung nur in wesentlichen Punkten geändert. ${ }^{6}$ Durch eine von der Interimsregierung eingesetzte Expertenkommission ausgearbeitet, trat das geänderte Grundgesetz im Sommer 1992 in Kraft. Die Grundstruktur des am britischen Westminstermodell orientierten parla-

6

Pretzell, Klaus, Grundlagen der Demokratie in der Verfassung Thailands (Teil 1), in: Südostasien aktuell, Januar 1994, S. 64-69: 64. 
mentarischen Zweikammersystems im Rahmen der konstitutionellen Monarchie blieb erhalten. Zentrale Bestimmungen, die in der Vergangenheit der Sicherung des Machteinflusses des Militärs gedient hatten, wurden jedoch revidiert. Die wichtigsten auf die Stellung des weiterhin ernannten Senats bezogenen Reformen zielten, erstens, auf die Schwächung des Senats zugunsten des Repräsentantenhauses. Der vom König ernannte Senat verfügte lediglich noch über ein suspensives (aufschiebendes) Veto gegenüber Gesetzesbeschlüssen des Repräsentantenhauses, das Gesetzesinitiativrecht wurde ihm entzogen. Seine Mitwirkungsrechte bei künftigen Verfassungsänderungen blieben gleichwohl erhalten. Damit sicherten sich insbesondere das Militär und dessen zivile Sympathisanten zunächst eine wichtige Vetoposition. Zweitens wurde die Stellung des Premier gegenüber Militär und ziviler Bürokratie gestärkt, indem das Vorschlagsrecht zur Ernennung der Senatsmitglieder auf den vom Vertrauen des Repräsentantenhauses abhängigen Premierminister überging.

\subsection{Die Stellung des Senats in der neuen Verfassung}

Zunächst gelang es den alten staatlichen Eliten noch, signifikante Machtressourcen zu erhalten, zumal das Militär seine institutionelle Autonomie und wichtige politische Vorrechte zumindest abgeschwächt bewahren konnte. ${ }^{7}$ Wie die folgende Übersicht zeigt, bildete der Senat auch nach 1992 die Kammer der Bürokraten und Militärs:

Tabelle 2: Durchschnittlicher Anteil ziviler Bürokraten und Militärs an den Mandaten (Senat, 1992-2000)

\begin{tabular}{|l|c|c|}
\hline & \multicolumn{2}{|c|}{ Zusammensetzung des Senats } \\
\hline & 1992-1996 $^{\mathbf{1}}$ & $\mathbf{1 9 9 6 - 2 0 0 0}$ \\
\hline Militärs & 37 & 19,6 \\
\hline Zivile Staatsbedienstete & 11 & 30,3 \\
\hline Bürokraten insgesamt & 75 & 49,9 \\
\hline
\end{tabular}

1 Die ersten beiden Spalten weisen für 1992-96 nur aktive Militärs und Staatsbeamte aus, während die Gesamtzahl auch ehemalige Amtsträger einschließt. Vennewald, Werner (Hrsg.), Demokratisierung und politischer Wandel, Münster / Hamburg, 1994, S. 215-262: 248 f. 
Quelle: Panitan Wattanayagorn, Thailand. The Elite's Shifting Conceptions of Security, in: Alagappa, Muthiah (ed.), Asian Security Practice. Material and Ideational Influences, Stanford, 1998, S. 417 - 444 (Fn 26); Surachart Bamrungsuk, From Dominance to Power Sharing The Military and Politics in Thailand, 1973-1992, unveröffentlichte Doktorarbeit, Columbia University 1999, S. 126; Bangkok Post, 24.031996.

Als zentraler Legitimationsparameter für die Aufrechterhaltung der Veto-Institution Senat diente der Verweis auf die angeblich oder tatsächlich niedrige Qualität der gewählten Amtsträger im Repräsentantenhaus. Ihnen wurde und wird im politischen Diskurs Thailands nicht nur von Seiten der ehemaligen Machteliten pauschal Unfähigkeit, Korruption und eine zweifelhafte Moral vorgeworfen, allesamt Eigenschaften, die (angeblich) eine "überparteiliche" Kontrolle durch einen "unpolitischen" Senat erforderten. ${ }^{8}$ Innerhalb der von zahlreichen reformorientierten Gruppierungen und zivilgesellschaftlichen Organisationen getragenen politischen Reformbewegung der 90er Jahre wurde diese negative Sicht politischer Parteien und ziviler Politiker zunehmend geteilt. Unter dem Eindruck wachsender Unzufriedenheit der Bürger mit den politischen Praktiken der Parlamentarier kam es innerhalb der Zivilgesellschaft nach 1992 zu einer tiefgreifenden Delegitimation der etablierten politischen Akteure und Institutionen der repräsentativen Demokratie. Berichte über das ansteigende Niveau politischer Korruption, das Desinteresse der Parlamentarier an politischen Fragen von nationaler Bedeutung, die Passivität des Parlaments im Bereich der Gesetzgebung und die Reformfeindlichkeit der etablierten Parteien, die Unfähigkeit der zwischen 1995 und 1997 amtierenden Regierungen Banharn und Chavalit sowie die programmatische Konturlosigkeit der thailändischen Parteien ${ }^{9}$ führten zu einem Meinungsklima, das von der thailändischen Sozialwissenschaftlerin Pasuk Phongpaichit folgendermaßen beschrieben wurde:

"In the view of [civil society], the parliamentary system had simply been coopted into the bureaucratic state. The battle was still between the people and the state, the people and paternalist domination, the people and rabop upatham, the patronage system which now encompassed not only bureaucrats but elected representatives. [...] With this declining faith in 'democracy' as the route to a better political future, and in parliamen-

McCargo, Duncan, Alternative Meanings of Political Reform in Contemporary Thailand, in: The Copenhagen Journal of Asian Studies 13 (1998), S. 5-59.

Vgl. hierzu allgemein Ammar Siamwalla, Can a Developing Democracy Manage Its Macroeconomy? The Case of Thailand, in: TDRI (ed.), Thailand's Boom and Bust, Bangkok, 1997, S. $53-$ 63; Anusorn Limmanee, Thailand, in: Sachsenröder, Wolfgang / Frings, Ulrike (eds.), Political Party and Democratic Development in East and Southeast Asia, Volume I: Southeast Asia, Aldershot, 1998, S. 403-448. 
tary institutions as a mechanism of change, the idea of 'civil society' has been seized upon to play the [role] as the repository of hope". ${ }^{10}$

Eines der zentralen Anliegen der Verfassungsreform von 1997 - der zweiten, verspäteten Phase der Verfassungsgebung der thailändischen Demokratie - war es folglich, die verbliebenen institutionellen Vetopositionen von Militär und Bürokratie durch die Neupositionierung des Senats im Gewaltengefüge des thailändischen Regierungssystem abzubauen und gleichzeitig die Kontrollfunktion der Kammer gegenüber dem Repräsentantenhaus und den politischen Parteien zu stärken. Während ein erster Anlauf zur Verfassungsreform 1994 noch am Widerstand der alten politischen Elite in Senat, Teilen des Repräsentantenhauses und der Regierung scheiterte, kam es im September 1996 zur Einrichtung einer verfassungsgebenden Versammlung (Constitutional Draft Assembly, CDA), auf deren Zusammensetzung die etablierten politischen Kräfte einschließlich des Senats nur geringen Einfluß hatten. ${ }^{11}$ Der von der CDA ausgearbeitete Verfassungsentwurf wurde der gemeinsamen Versammlung beider Häuser des Parlaments (Nationalversammlung) zur Entscheidung vorgelegt. Obwohl der Entwurf klar gegen die vested interests in der staatlichen Verwaltung, dem Militär und dem Repräsentantenhaus gerichtet war, stimmten die Abgeordneten mit großer Mehrheit für die Annahme der Verfassung. Angesichts der eng begrenzten Entscheidungsoptionen der Versammlung - der Entwurf mußte ohne Änderung angenommen oder insgesamt verworfen werden - und des starken politischen Drucks, der von gesellschaftlichen Gruppen und den Medien auf die Nationalversammlung ausgeübt wurde, sowie nicht zuletzt aufgrund des innenpolitischen Meinungsklimas auf dem Höhepunkt der Währungskrise im Spätsommer 1997 blieb den etablierten politischen Machtträgern kaum eine andere Entscheidungsalternative. Die neue Verfassung trat schließlich am 11. Oktober 1997 in Kraft. $^{12}$

In unserem Zusammenhang interessieren jene konstitutionellen Reformen, die sich auf die Stellung des Senats beziehen. In ihrer Konsequenz zielen sie auf eine verfassungsrechtliche Neupositionierung der zweiten Kammer innerhalb des Institutionengefüges der thailändischen Demokratie, die sich in Anlehnung an den amerikanischen Verfassungstheoretiker

Für eine kritische Bestandsaufnahme der Rolle der thailändischen Presse in diesem Prozeß, vgl. McCargo, Duncan, The International Media and the Domestic Political Coverage of the Thai Press, in: Modern Asian Studies 33 (1999), S. 551-579.

Suchit, Bunbongkarn, Thailand's Successful Reforms, in: Journal of Democracy 10 (1998), Nr. 4, S. 54-68: 59 f.; ausführlich: Borwornsak, Uwanno / Burns, Wayne D., The Thai Constitution of 1997: Sources and Process, in: University of British Columbia Law Review 32 (1998), Nr. 2, S. 227-249.

12

Constitution of the Kingdom of Thailand 1997, Office of the Council of State, Bangkok, 1997. 
Bruce Ackerman ${ }^{13}$ als Entwicklung von einer klassischen Vetokammer zu einer "deliberativen zweiten Kammer" der Demokratie bezeichnet läßt. Ziel der verfassungsgebenden Versammlung war es, dem Senat den Status einer von politischen Parteien und Interessengruppen unabhängigen, in erster Linie kontrollierend und beratend tätig werden Kammer zuzuweisen. Dies läßt sich vor allem an zwei Kriterien aufzeigen:

1. die verfassungsrechtliche Kompetenzzuweisung an den Senat sowie

2. die für repräsentative Demokratien ungewöhnlichen Kandidaturbestimmungen. Sie konstruieren nicht nur eine organschaftliche Trennung zwischen Senat, Repräsentantenhaus und Regierung, sondern zielen auch auf eine strikte organisatorische Trennung von staatlicher Verwaltung und Militär sowie den politischen Parteien einerseits und dem Senat andererseits.

ad 1) Weiterhin ist der Senat mit Blick auf seine parlamentarischen Kernkompetenzen dem Repräsentantenhaus nachgeordnet: Weder verfügt die zweite Kammer über das Gesetzesinitiativrecht, noch hat sie Einfluß auf das Zustandekommen oder die Abwahl der Regierung. Im Bereich der Verfassungsgebung (Art. 313) bilden beide Kammern gemeinsam die Nationalversammlung, die ihrerseits mit absoluter Mehrheit über verfassungsändernde Gesetze beschließt. Im Vergleich zu einem Verfahren, in dem beide Kammern getrennt über Verfassungsänderungen beschließen, resultiert hieraus eine geringere "Vetomacht" des Senats $^{14}$. In Anlehnung an Arend Lijphart ${ }^{15}$ entspricht das Zweikammersystem Thailands wie bisher eindeutig dem Typ des "asymmetrischen Bikameralismus".

Der Senat verfügt jedoch über eine Reihe teilweise neuer Kompetenzen, die ihn potentiell zu einem einflußreichen Organ machen. Zu nennen ist hier zunächst das Vetorecht des Senats, das es ihm ermöglicht, Gesetzesvorhaben bis zu 180 Tage zu blockieren (Art. 175). Ein Senatsveto kann vom Repräsentantenhaus nur mit absoluter Mehrheit seiner Mitglieder aufgehoben werden (Art. 176). Werden die maximal 60 Tage hinzugerechnet, welche dem Senat zur Beratung eines vom Repräsentantenhaus beschlossenen Gesetzes zur Verfügung

Ackerman, Bruce, The New Separation of Powers, in: Harvard Law Review 113 (2000), Nr. 3, S. 633-730: 684.

Pasuk, Phongpaichit, Civilising the State: State, Civil Society and Politics in Thailand. The Wertheim Lecture 1999, Center of Asian Studies, Amsterdam, 1999, S. 11 f.; Verfassungsänderungen sind in drei Lesungen von der Nationalversammlung zu behandeln. Während die 500 Mitglieder des Repräsentantenhauses sowie die 200 Mitglieder des Senats in erster und dritter Lesung jeweils mit absoluter Mehrheit aller Mitglieder der Nationalversammlung zustimmen müssen, genügt bei der zweiten Abstimmung die relative Mehrheit. Selbst bei geschlossenem Abstimmungsverhalten seiner Mitglieder ist der Senat daher nicht in der Lage, ein geschlossen auftretendes Repräsentantenhaus zu blockieren.

Lijphart, Arend, Patterns of Democracy. Government Forms and Performance in thirty-six Countries, New Haven / London, 1999. 
stehen - bei ausgabewirksamen Gesetzen 30 Tage (Art. 174) - so ergibt sich hieraus ein Verzögerungszeitraum von maximal sieben Monaten. Des weiteren kann der Senat Änderungen zu einem vom Repräsentantenhaus bereits verabschiedeten Gesetzen beschließen (Art. 175). Stimmt das Repräsentantenhaus der geänderten Fassung nicht zu, muß nach Ablauf von sechs Monaten erneut über das betroffene Gesetz beschlossen werden (Art. 176). Des weiteren tritt der Senat im Impeachment-Verfahren gegen Mitglieder der Regierung, des Repräsentantenhauses, anderer Verfassungsorgane sowie gegen hohe Beamte als Gerichtshof in Erscheinung. Schließlich bildet der Senat das Wahl- bzw. Designationsorgan bei der Besetzung der National Counter Corruption Commission, der Nationalen Menschenrechtskommission, der Wahlkommission, des Amts der Ombudsmänner sowie des Verfassungs- und des obersten Verwaltungsgerichtshofs.

Besondere Bedeutung für die Kontrollfunktion des Senats hat seine Stellung als Gericht im Amtsenthebungsverfahren. Diese, aus institutionenhistorischer Perspektive klassische Funktion einer zweiten Kammer ist für sich betrachtet nicht ungewöhnlich. Wie die Erfahrungen anderer Demokratien, in denen der Senat über vergleichbare Rechte verfügt (Frankreich, USA), zeigen, ist dieses Recht für das alltägliche Funktionieren des politischen Systems kaum von Bedeutung. Im thailändischen Kontext kommt ihm aber besondere Relevanz zu, da der Antrag auf Einleitung eines Amtsenthebungsverfahrens nicht vom Parlament, sondern auf plebiszitären Wege im Rahmen einer Petitionskampagne durch den Wähler gestellt werden kann, sofern 50.000 Wahlberechtigte mit ihrer Unterschrift einen solchen Antrag unterstützen. Nach Ansicht thailändischer Sozialwissenschaftler handelt es sich hierbei um "the most innovative, most dramatic, and most feared provision in the new charter". ${ }^{16}$ Während die Initiative für ein Amtsenthebungsverfahren beim Wähler liegt, erfolgt die Entscheidung zur Einleitung des Verfahrens sowie die Verfahrensführung durch den Senat. Angesichts des bekanntermaßen sehr hohen Korruptionsniveaus in Regierung, Verwaltung und Parlament ist dem Senat hiermit, den politischen Willen zur Verfahrenseröffnung vorausgesetzt, ein machtvolles Instrument zur Disziplinierung und Kontrolle der konkurrierenden Gewalten an die Hand gegeben worden.

ad 2) Die neue Verfassung setzt an die Stelle des vormals ernannten Oberhauses eine frei gewählte zweite Kammer. Die Direktwahl soll garantieren, daß der Senat zukünftig weder von Vertretern der alten politischen Elite aus Militär und Bürokratie, noch von den politischen Parteien und ihnen nahestehenden Unternehmerkreisen dominiert wird, sondern von "überparteilichen" und unabhängig handelnden Persönlichkeiten aus allen Teilen der Gesellschaft als wirksames Kontroll- und Beratungsorgan genutzt werden kann. Um dieses Ziel zu erreichen, wurden in der Verfassung ausgesprochen strikte Beschränkungen der Kandidaturmöglichkeiten festgelegt. So sind amtierende Mitglieder des Repräsentantenhau- 
ses ebenso von der Kandidatur ausgeschlossen (Art. 126), wie Mitglieder der staatlichen Verwaltung, Beschäftigte von Staatsunternehmen, Angehörige der sechs Verfassungsorgane sowie amtierende Senatoren (Art. 109). Vor allem aber sind Mitglieder politischer Parteien von der Kandidatur zum Senat ausgeschlossen, während gleichzeitig die Mitgliedschaft in einer Partei Voraussetzung für die Kandidatur zum Repräsentantenhaus ist (Art. 107 Abs. 4, Art. 126 Abs. 1).

Gerade in dieser Bestimmung zeigt sich das geringe Ansehen politischer Parteien in der thailändischen Politik, wie es bereits in den 15 Vorläuferinnen der jetzigen Verfassung zum Ausdruck kam. So hat der thailändische Politikwissenschaftler Anusorn Limmanee in diesem Zusammenhang treffend festgestellt:

"This obviously reflects the relatively low status of political parties, at least in the eyes of most members of the previous constitution-drafting committee, if not the Thai public in general. It means that political parties have not yet been generally accepted or fully institutionalized in this society. The status of political aliens leads to successive attempts by political authorities to regulate activities of parties for fear of their possible threats to social order." ${ }^{17}$

Hervorzuheben ist, daß die Verfassung zwar die Bedeutung politischer Parteien für das demokratische System anerkennt, da ausschließlich Parteien Kandidaten für die Wahl zum Repräsentantenhaus aufstellen können. Zugleich sucht man in der Verfassung jedoch vergeblich nach einer positiven Funktionsbeschreibung der politischen Parteien. Vielmehr enthält sie lediglich Verbotsbestimmungen für Parteien und deren Mitglieder und definiert den verfassungsrechtlichen Status der Parteien ausschließlich negativ. Gleiches gilt für das Wahlgesetz zum Repräsentantenhaus und Senat sowie für das neue Parteiengesetz vom Juni $1998^{18}$. Verfassung und Parteien- sowie Wahlgesetz sind somit institutioneller Ausdruck einer politischen Reformbewegung, welche versucht hat, die unbestreitbar problematische Wirkung politischer Parteien auf die Entwicklung der thailändischen Demokratie in den 90er Jahren durch Neutralisierung ihres Einflusses im parlamentarischen System zu bekämpfen. Überlegungen, durch ein neues Parteiengesetz die innere Demokratisierung der Parteien zu fördern, um die repräsentative Demokratie aus der Parteienverdrossenheit durch die politischen Skandale der 90er Jahre heraus zu führen, hatten angesichts der sprichwörtlichen Abkehr der (Zivil-)Gesellschaft von den zentralen Akteuren der repräsentativen Demokratie im Reformdiskurs des letzten Jahrzehnts kaum eine Chance.

Organic Law on Political Parties (ParteienG), veröffentlicht in Government Gazette, vol. 115, Part 35a, 9. Juni 1998, vorläufige Übersetzung. Ich danke Dr. Norbert Eschborn, Bangkok, für die Bereitstellung der inoffiziellen Übersetzungen dieser Gesetze in englischer Sprache. 
Zugleich konstruieren die Verfassungsartikel 106, 107, 109 sowie Art. 125 einen stark exklusiven demos: Alle Personen ohne tertiären Bildungsabschluß, buddhistische Mönche sowie alle Staatsbediensteten (was auch die in Staatsbetrieben Beschäftigten einschließt) sind von der Kandidatur für den Senat und das Repräsentantenhaus ausgeschlossen (Art. 107, 109, 125). Diese mit dem erklärten Ziel der Verbesserung der intellektuellen und moralischen Qualität von Mandatsträgern, der Trennung von Staat und "Kirche" bzw. der Beschneidung des politischen Einflusses des Militärs begründeten Bestimmungen - paradoxer Weise auf breite Zustimmung in der Bevölkerung stoßend ${ }^{19}$ - berauben schätzungsweise $96,6 \%$ der Wahlberechtigten ihres passiven Wahlrechts ${ }^{20}$. Bedenklich erscheint jedoch nicht nur der Ausschluß breiter Bevölkerungsgruppen an sich, sondern auch das Motiv dieser Regelung. Nach Aussagen von Mitgliedern der Constitutional Development Assembly war es ein implizites Ziel, hierdurch den Einfluß des organisierten Verbrechens auf die nationale Politik, der in den letzten beiden Jahrzehnten durch die direkte Kandidatur von jao pho ${ }^{21}$ (lokaler Bosse, "Paten") oder deren finanzielle Unterstützung für Kandidaten stark zunahm, ${ }^{22}$ zu beschneiden ${ }^{23}$. Zum einen soll hier also die Verfassung gezielt für den Ausschluß bestimmter "Einflußgruppen" genutzt werden; zum anderen ist es angesichts

In zahlreichen öffentlichen Anhörungen und ca. 800.000 Eingaben, welche die Arbeit der verfassungsgebenden Versammlung begleiteten, war eine breite Zustimmung und sogar der Wunsch nach Einführung der Bachelor-Klausel erkennbar (Interviews mit Prof. Borwornsak, seinerzeit Secretary of the Constitution Scrutiny Committee des House of Representatives, 31.8.1999 und Prof. Amara, Mitglied der CDA, 2.9.1999).

Eigene Berechnungen auf der Grundlage des Anteils der Personen mit Universitätsabschluß an der ökonomisch aktiven Bevölkerung (1990) sowie der registrierten Wähler (November 1996; Angaben in: Reinecke, Gerd, Thailand - die gefährdete Demokratie, in: Schubert, Gunter / Tetzlaff, Rainer / Vennewald, Werner (Hrsg.), Demokratisierung und politischer Wandel, Münster / Hamburg 1994, S. 215-262: 242; ILO 1993, S. 32; Statistical Yearbook Thailand, 1996, S. 443 ff.).

"The term conveys not only wealth and power but also an ability to operate above the Law. Most [jao pho] are ethnic Chinese by origin and generally based in the provinces. They have wide business interests, covering both legitimate and criminal activities. [...] They move closely with powerful bureaucrats, policemen and military figures. They sit in positions of authority in local administration. They play a key role in parliamentary elections. [...] In the context of present-day Thailand the term is used to refer to an influential person who can use his wealth and informal power (through patronage, bribery, violence or other means) to put himself above the Law and to provide extra-legal protection for others." Vgl. Pasuk, Phongpaichit / Sungsidh, Piriyarangsan / Nualnoi, Treerat, Guns, Girls, Gambling, Ganja. Thailand's Illegal Economy and Public Policy, Chiang Mai, 1998, S. 57, 59.

Sombat, Chantornuvong, Local Godfathers in Thai Politics: A Preliminary Observation. Paper presented for the 5th International Conference on Thai Studies, London, 1993; Albritton, Robert $B$., Political Parties and Elections in Thailand in an Era of Globalization: No longer a SemiDemocracy. Proceedings of the 6th International Conference on Thai Studies, Chiang Mai, 14 - 17 October, 1996, S. 11. 
der weitverbreiteten Korruption im Hochschulsystem Thailands zweifelhaft, ob die Regelung nicht einfach durch "Kauf" eines Hochschulabschlusses umgangen werden kann.

\section{Organisatorische Rahmenbedingungen der Wahl}

Den organisatorischen Rahmen für die Wahl der insgesamt 200 (vormals 260) Senatoren bildeten die Bestimmungen der Verfassung, das von der Wahlkommission ausgearbeitete neue Wahlgesetz $^{24}$ (WahlG) ${ }^{25}$ sowie das Gesetz über die Wahlkommission (WahlKomG). ${ }^{26}$ Art. 122 der neuen thailändischen Verfassung legt fest, daß jede der 76 Provinzen des Landes für die Wahl zum Senat einen Wahlkreis bildet. Die Anzahl der pro Provinz vergebenen Mandate bestimmt sich im wesentlichen nach der Zahl ihrer registrierten Wähler. In einem ersten Schritt wurde für je 150.000 Wähler ein Mandat pro Provinz vergeben. Die Zahl der Mandate pro Provinz lag zwischen einem und achtzehn. Als Entscheidungsregel dient die relative Mehrheit der abgegebenen, gültigen Stimmen (Art. 93 WahlG). Entsprechend läßt sich das Wahlsystem als kombiniertes Mehrheitswahlsystem mit Elementen der relativen Mehrheitswahl in Einmannwahlkreisen, binominaler Mehrheitswahl und SingleNon-Transferable Vote System bezeichnen.

Zuständig für die Durchführung der Wahlen war die im November 1997 eingerichtete Nationale Wahlkommission (Election Commission, EC). Das von der Regierung unabhängige Verfassungsorgan übernahm vom bislang zuständigen Innenministerium die Aufgabe der Wahlorganisation und -durchführung (Art. 10 WahlKomG $^{27}$ ). Die Schaffung einer unabhängigen Wahlkommission hatte zum Ziel, die bei früheren Wahlen gängigen Praktiken der Wahlkreismanipulation sowie politisch motivierter Unregelmäßigkeiten bei der Stimmenauszählung abzustellen. ${ }^{28}$ Um den "überparteilichen" Charakter der Wahlen und möglichst gleiche Wettbewerbschancen zwischen den Kandidaten zu gewährleisten, war den Bewerbern die Abhaltung von politischen Veranstaltungen oder anderen öffentlichen Auftritten zur Eigenwerbung untersagt. Verboten waren auch Kontakte zu politischen Mandatsträger, Parteien und staatlichen Behörden sofern sie der eigenen Kandidatur dienten sowie die Beschäftigung von Wahlhelfern (Art. 36 ParteienG, Art. 91 f. WahlG). Die

Organic Law on the Election of Members of the House of Representatives and Senators (WahlG), veröffentlicht in Government Gazette, vol. 115, Part 35a, 9. Juni 1998, vorläufige Übersetzung.

25

26

27

28

Letzteres gilt auch für die spätestens Ende diesen Jahres fälligen Wahl zum Repräsentantenhaus.

Organic Law on the Election Commission (WahlKomG), veröffentlicht in Government Gazette, vol. 115, Part 35a, 9. Juni 1998, vorläufige Übersetzung.

Organic Law on the Election Commission (WahlKomG), veröffentlicht in Government Gazette, vol. 115, Part 35a, 9. Juni 1998, vorläufige Übersetzung.

Vgl. Anusorn (Fn. 10), S. 437. 
einzige erlaubte Art der Wahlwerbung beschränkte sich auf die Verbreitung von "Informationsplakaten", auf denen lediglich Name und Beruf des Bewerbers sowie dessen Kandidatennummer genannt werden durften. ${ }^{29}$ Angesichts dieser ausgesprochen restriktiven und für Wahlen in repräsentativen Demokratien sehr ungewöhnlichen Bestimmungen markierten die Wahlen eine besondere Herausforderung für noch junge Wahlkommission.

Tabelle 3: Wahlkreiseinteilung und Wahlkreisgröße

\begin{tabular}{|l|c|c|c|}
\hline \multicolumn{1}{|c|}{ Wahlkreise } & $\begin{array}{c}\text { Anzahl der } \\
\text { Wahlkreise }\end{array}$ & \multicolumn{2}{|c|}{ Durchschnittliche } \\
& & Wählerzahl* & Ratio Wähler/Mandate* \\
\hline Einmannwahlkreisen & 22 & 212 & 212 \\
\hline Zweimannwahlkreisen & 26 & 414 & 207 \\
\hline SNTV & 28 & & \\
Davon Wahlkreise mit & & & 219 \\
3 Mandaten & 13 & 659 & 215 \\
4 Mandaten & 6 & 861 & 206 \\
5 Mandaten & 5 & 1.033 & 226 \\
6 Mandaten & 2 & 1.204 & 213 \\
8 Mandaten & 1 & 1.815 & \\
18 Mandaten & 1 & 3.848 & \\
\hline
\end{tabular}

* Angaben in Tausend Wählern.

Quelle: Bangkok Post, 2. März 2000 und eigene Berechnungen nach Angaben der Nationalen Wahlkommission (http://202.183.254.190/report/show004.asp; in Thai).

Deren Entschlossenheit, ihrem Verfassungsauftrag nachzukommen, zeigte sich bereits im Vorfeld der Wahl, als erstmals in der thailändischen Geschichte Bewerbern aufgrund von Verstößen gegen das Wahlgesetz die Zulassung zur Kandidatur verweigert wurde. Hiervon betroffen waren insgesamt etwa 30 Kandidaten. ${ }^{30}$ Bereits vor der Wahl wurden 35 Kandidaten von der Wahlkommission aufgrund des Verdachts auf Stimmenkauf und Vorlage gefälschter Kandidaturunterlagen "vorgemerkt" (backlisted), was einer Ankündigung gleichkam, im Falle ihres Wahlsiegs das Ergebnis auf seine Gültigkeit hin zu überjedoch geduldeten Plakate durfte das Zehnfache der Wahlstationen pro Provinz nicht überschreiten. Für Bangkok bedeutete dies eine Zahl von knapp 60.000 Plakaten pro Kandidat.

The Nation, 22.12.1999; Bangkok Post, 2.3.2000. 
prüfen. ${ }^{31}$ Gleichwohl deutete sich in diesem Zusammenhang bereits an, daß die Einhaltung der zahlreichen Beschränkungen, denen Kandidatur und Wahlwerbung unterlagen, kaum zu gewährleisten war, zumal einige Bestimmungen - so etwa die Definition der laut Wahlgesetz von der Kandidatur ausgeschlossenen Bewerber - wenig konkret formuliert waren, so daß ihre Auslegung fast zwangsläufig umstritten war.

\section{Die Durchführung der Wahl}

Bei der Wahl am 4. März diesen Jahres bewarben sich insgesamt 1.522 Kandidaten um 200 Mandate, alleine in Bangkok stellten sich 264 Bewerber der Wählerentscheidung. ${ }^{32}$ Dies entsprach national einer Quote von 7,6 Kandidaten pro Mandat, in Bangkok lag sie mit 14,6 am höchsten. Die Wahlbeteiligung betrug im ersten Wahlgang landesweit 71,9\%, in der Hauptstadt erreichte sie $71,74 \% .{ }^{33}$ Während in der Vergangenheit im Nordosten, der ärmsten und am wenigsten entwickelten Region des Landes, die Wahlbeteiligung besonders hoch lag, wies diese Region diesmal die niedrigste Partizipationsrate auf. In der Hauptstadt wiederum markierte die Wahl eine Rekordbeteiligung. Die landesweit und insbesondere in Bangkok sehr hohe Wahlbeteiligung war sicherlich auch der neu eingeführten Wahlpflicht geschuldet. Vor allem in der Hauptstadt war sie aber auch das Ergebnis einer erfolgreichen Mobilisierungskampagne zahlreicher NGOs und zivilgesellschaftlicher Gruppen im Zusammenwirken mit den thailändischen Medien. Mit Blick auf die vergleichsweise geringe Beteiligung im Nordosten kann vermutet werden, daß die strikten Wahlkampfbestimmungen und die starke Präsenz unabhängiger Wahlbeobachter sowie die Durchführung der Wahl durch eine unabhängige Kommission die Mobilisierung der Wählerschaft durch Kandidaten (Stimmenkauf) und die lokalen Behörden zumindest erschwerten. Diese beiden Faktoren wurden jedoch in der Vergangenheit von manchen Beobachtern als Hauptursache für die traditionell hohe Wahlbeteiligung in der Region genannt. ${ }^{34}$

Zunächst kann festgestellt werden, daß politisch motivierte Gewalt nur in geringerem Umfange und vor allem in den "Problemprovinzen" im Nordosten vorkam. Als problematisch erwiesen sich vielmehr wie bereits bei früheren Wahlen andere Formen der illegalen Beeinflussung von Organisation und Ablauf der Wahlen. So wurden auch diesmal Fäl-

The Nation, 3. März 2000, S. A1.

Asian Wall Street Journal, 3.-4.03.2000, S. 7.

33

The Nation, 9.3.2000.

34

Vgl. Prasert, Ragthaidee, A Comparative Analysis of Voters in the South and Northeast Regions, Bangkok 1989; Anusorn Limmanee, Political Business Cycles in Thailand, 1979 - 1992: General Election and Currency Circulation, Research Report Submitted to the Institute of Thai Studies, Chulalongkorn University, Bangkok, 1995 und ders. (Fn. 10). 
schungen von Wählerregistern und Stimmzetteln, das Austauschen von Wahlurnen und die Manipulation der Stimmenauszählung beobachtet, lediglich in 15 von 76 Provinzen lagen

Tabelle 4: Wahlbeteiligung in den Regionen

\begin{tabular}{|l|l|l|l|l|l|l|l|}
\hline Region & $\mathbf{1 9 8 6}$ & $\mathbf{1 9 8 8}$ & $\mathbf{1 9 9 2 / 1}$ & $\mathbf{1 9 9 2 / 2}$ & $\mathbf{1 9 9 5}$ & $\mathbf{1 9 9 6}$ & $\mathbf{2 0 0 0 / 1}$ \\
\hline Bangkok & 38,13 & 37,5 & 42,57 & 47,40 & 49,82 & 48,97 & $\mathbf{7 1 , 7 4}$ \\
\hline Zentral $^{2}$ & 57,29 & 58,57 & 56,59 & 58,80 & 62,62 & 63,41 & $\mathbf{7 5 , 2 7}$ \\
\hline Ost & 55,47 & 57,77 & 55,73 & 58,95 & 62,89 & 66,14 & $\mathbf{6 9 , 9 8}$ \\
\hline West & 57,24 & 56,03 & 55,86 & 59,15 & 65,78 & 68,33 & $\mathbf{7 4 , 6 2}$ \\
\hline Nord & 65,75 & 69,10 & 62,24 & 63,91 & 64,26 & 65,58 & $\mathbf{7 3 , 3 0}$ \\
\hline Nordost & 73,72 & 77,64 & 64,81 & 67,77 & 63,16 & 63,16 & $\mathbf{6 8 , 0 0}$ \\
\hline Süd & 61,01 & 63,81 & 58,94 & 60,11 & 63,43 & 60,89 & $\mathbf{7 6 , 5 1}$ \\
\hline National & 61,43 & 63,56 & 59,24 & 61,59 & 62,04 & 62,41 & $\mathbf{7 1 , 9 0}$ \\
\hline
\end{tabular}

1 Erster Wahlgang

2 Einschließlich Nakhon Pathom, Nonthaburi, Pathum Thani, Samut Prakan und Samut Sakhon

Quelle: Eigene Berechnungen nach Angaben in: National Statistical Office, Statistical Yearbook Thailand, verschiedene Jahrgänge, Bankok (1986-1996) und Election Commission (http:// 202.183.254.190/report/show002.asp, besucht am 19.4.2000; in Thai). Die Wahlbeteligung sank im zweiten Wahlgang auf landesweit 53\% ab (The Nation, http://www. bangkokpost. net/today/020 500News15.html; besucht am 02.05.2000).

keine Berichte hierüber vor (The Nation, 5. März 2000: A1). Obwohl keine systematische Angaben verfügbar sind, legen zahlreiche Berichte privater Organisationen (PollWatch, People's Network for Election in Thailand, Law Society of Thailand) wie auch der Wahlkommission den Schluß nahe, daß die etablierte Betrugsstruktur thailändischer Wahlen erneut, wenn auch in geringem Umfange als sonst, ihre Funktion entfaltete. Die Hoffnung des Verfassungsgebers, mit der Einführung einer unabhängigen Wahlkommission Unregelmäßigkeiten im Vorfeld und während der Wahlen besser kontrollieren zu können, erfüllte sich nur bedingt. Wie Mitglieder der Wahlkommission selbst zugaben, war die Einhaltung der restriktiven Wahlkampfbestimmungen, insbesondere des Verbots parteipolitischer Bindungen der Kandidaten sowie des Stimmenkaufs, kaum zu gewährleisten. ${ }^{35}$ Als problematisch erwies sich vor allem die unzureichende Institutionalisierung und, damit 
verbunden, mangelnde eingeschränkte Funktionsfähigkeit die Wahlbehörde auf nationaler als auch auf lokaler Ebene. Sowohl bei der Personalausstattung als auch hinsichtlich ihrer technischen Infrastruktur war die Wahlbehörde massiv auf die Mithilfe anderer Ministerien und der lokalen Verwaltungen angewiesen. Als hinderlich erwies sich insbesondere das unzureichende Budget der Wahlbehörde ${ }^{36}$. In Bangkok mussten sich zahlreiche Wahlbüros regelrecht verschulden, um die Kosten des ersten Wahlgangs bestreiten zu können. ${ }^{37}$ Bis zum Nachmittag des Wahltages lagen der Wahlbehörde sowie verschiedenen Organisationen zur Wahlbeobachtung Meldungen über mehr als 1.000 Verstöße gegen das Wahlgesetz vor.

Tabelle 5: Verstöße gegen das Wahlgesetz, Thailand 1992-2000

\begin{tabular}{|l|c|}
\hline & Gemeldete Verstöße gegen das Wahlgesetz \\
\hline September 1992 (R) & 2.500 \\
\hline Juli 1995 (R) & 3.119 \\
\hline November 1996 (R) & 4.260 \\
\hline März 2000 (S, 1. Wahlgang) & 1.120 \\
\hline April 2000 (S, 2. Wahlgang) & 501 \\
\hline
\end{tabular}

Quelle: King, Daniel E., The Thai Parliamentary Elections of 1992, in: Electoral Studies 12 (1993), S. 268-274: 272; Anusorn (Fn. 9), S. 436; The Nation 5.3.2000, S. A4; The Nation 1.5.2000 (http://nationmultimedia.com/new/29po03.shtml, besucht am 01.05.2000); Bangkok Post, 30.4.2000 (http://www.bangkokpost.net/today/30400_News001.html, besucht am 30.04. 2000).

Einschätzungen politischer Beobachter zufolge hatten ca. 70\% der Kandidaten enge Verbindungen zu politischen Parteien oder führenden Parteivertretern verschiedener politischen Richtungen und Lager, ${ }^{38}$ zahlreiche Familienangehörige von Regierungsmitgliedern oder Parlamentariern kandidierten als "Stellvertreter" ${ }^{39}$; Bürokratie und Militär stellten wie

So hat die Regierung unter dem Argument von Sparzwängen der EC im August 1999 lediglich etwa ein Drittel der beantragten Jahresmittel zugewiesen; bei den angeforderten Mitteln für Verwaltung, Personalausgaben und Projektimplementation bewilligte die Regierung gar nur rund 15\%; vgl. Bangkok Post, 19.8.1999 und 20.8.1999.

Bangkok Post, 9.3.2000 und 4.4.2000, S. 3; The Nation, 10. März 2000, S. A1.

38 Bangkok Post, 2.3.2000.

39

Bangkok Post, 6.3.2000; Eschborn, Norbert, Thailand vor dem Superwahljahr 2000. Probleme Personen - Perspektiven, in: KAS-Auslandsinformationen 10 (1999), S. 54-75; Helfen, Thomas, Politischer Kurzbericht. Thailand feiert den "runden" Geburtstag seines Königs - Rennen zu den 
zuvor bei der Auswahl im Ernennungsverfahren die größte Gruppe der Anwärter auf einen Senatssitz:

Tabelle 6: Karrierehintergrund der Kandidaten zur ersten Senatswahl (1. Wahlgang)*

\begin{tabular}{|l|c|c|}
\hline & National $^{\mathbf{1}}$ & Bangkok $^{\mathbf{2}}$ \\
\hline Aktive und Ehemalige Staatsbedienstete $^{\mathbf{3}}$ & 39,5 & $28,3^{5}(5,4)^{6}$ \\
\hline Juristen & 17,0 & 17,5 \\
\hline Unternehmer $^{\mathbf{4}}$ & 20,9 & 20,8 \\
\hline Ehemalige Parlamentarier u. Parteipolitiker & 1,3 & 10,8 \\
\hline Andere & 21,2 & 17,1 \\
\hline
\end{tabular}

* 4. März 2000; zum zweiten Wahlgang (29. April 2000) waren nur Kandidaten aus dem ersten Wahlgang zugelassen.

1 Insgesamt 1521 Kandidaten

2 Insgesamt 265 Kandidaten

3 einschließlich Hochschullehrer

4 einschließlich Handelsunternehmer

5 Staatsbedienstete;

6 Ehemalige Militärs.

Quelle: Nationale Anteile sind eigene Berechnungen nach Angaben in Bangkok Post vom 2. März 2000; Angaben für Bangkok sind eigene Berechnungen basierend auf Angaben für 240 Kandidaten in Bangkok Post vom 23. März 2000.

In fast der Hälfte alle Provinzen beeinträchtigten Verstöße gegen das Wahlgesetz den Ausgang der Wahlen so stark, daß sich die Wahlkommission entschloß, das Wahlergebnis in 13 Provinzen vollständig sowie in 22 weiteren Provinzen teilweise zu annullieren. ${ }^{40}$ Für 78

Senatswahlen hat begonnen - Regierung mit Mißtrauensdebatte konfrontiert (wysiwg://main.8/ 40 http://www.kas.de/international/laenderberichte/thailand_dez.html, vom 08. 12. 1999.

Bangkok Post, 28.4.2000. Laut Gesetz über die Wahlkommission kann die EC in einigen oder allen Wahlbezirken neue Wahlen anzusetzen, "when there occurs the convincing evidence that the election or the voting at a referendum in that are those polling stations have not proceeded in an honest and fair manner, under the rules and procedure prescribed by the Election Commission" (Art. 10 Abs. 7 WahlKomG, vgl. oben Fn. 26). Noch eindeutiger ist die Formulierung im Wahlgesetz: Reicht ein Wähler oder ein Kandidat bei der Wahlbehörde eine Petition ein, aus der hervorgeht, daß in seinem Wahlkreis Unregelmäßigkeiten bei der Wahl oder Verstöße gegen das Wahlgesetz vorgelegen haben und wenn die Wahlbehörde zu der Einschätzung gelangt "that the election in any polling station or in any constituency has been dishonest and unjust and that a new counting of votes or a new election should be held, the Election Commission shall order for a new vote-counting or a new election in that polling station or that constituency. In the case of the elec- 
der insgesamt 200 Mandate wurde für den 29. April ein zweiter Wahlgang angesetzt. Wie bei allen Wahlen seit der Demokratisierung war die korrekte Durchführung am stärksten im Nordosten des Landes beeinträchtigt, was deshalb besonders ins Gewicht fiel, da diese Region die meisten Wähler und Abgeordneten stellt. Über die Hälfte aller annullierten Wahlsieger waren im Nordosten angetreten, für fast drei Viertel der in der Region vergebenen Sitze mußte erneut gewählt werden. Im Osten und der Zentralregion des Landes wurde jeweils die Hälfte der zu vergebenden Senatssitze erneut zur Wahl ausgeschrieben:

Tabelle 7: Annullierte Wahlsiege nach Regionen

\begin{tabular}{|l|c|c|c|c|c|c|}
\hline \multicolumn{1}{|c|}{ Region } & Wahlberechtigte & \multicolumn{2}{c|}{$\begin{array}{r}\text { Senatssitze } \\
\text { (A) }\end{array}$} & \multicolumn{2}{c|}{$\begin{array}{c}\text { Annullierte } \\
\text { Sieger (B) }\end{array}$} & Anteil von \\
& Anteil & Absolut & Anteil & Absolut & Anteil & \\
\hline Bangkok & 9,4 & 18 & 9 & 2 & 11,5 & 11,1 \\
\hline Zentral & 11,0 & 20 & 10 & 5 & 12,8 & 50,0 \\
\hline Osten & 6,7 & 14 & 7 & 7 & 9,0 & 50,0 \\
\hline Westen & 5,88 & 11 & 5,5 & 1 & 1,3 & 9,1 \\
\hline Norden & 20,2 & 41 & 20,5 & 12 & 15,3 & 29,2 \\
\hline Nordosten & 34,3 & 69 & 34,5 & 46 & 59,0 & 71,8 \\
\hline Süden & 12,4 & 27 & 13,5 & 5 & 6,4 & 18,5 \\
\hline
\end{tabular}

Quelle: Eigene Berechnungen nach Angaben in: National Statistical Office, Statistical Yearbook Thailand 1996, Bangkok, 1996, S. 440-443; The Nation, 29.3.2000; Bangkok Post, 2.3.2000.

In mindestens 40 Provinzen kam es in großem Maßstab zu Stimmenkauf (Bangkok Post 5.3.2000). Die pro Stimme angebotenen Beträge lagen je nach Region zwischen 20 und 1000 Baht (ca. 1 bis 50 DM). ${ }^{41}$ Obwohl bei den Senatswahlen jegliche Form der Wahlwerbung verboten war, bezifferten hochrangige Mitglieder der für die Bekämpfung des Stimmenkaufs zuständigen Nationalen Polizeibehörde die Gesamthöhe der während der Wahlperiode und am Wahltag "investierten" Beträge auf etwa $1 \mathrm{Mrd} . \mathrm{DM}^{42}$. Wieviel davon für

tion of senators, the Election Commission may order for only the new counting of votes or the new election of the senator against whom the petition was made" (Art. 95 WahlG, oben Fn. 24).

Vgl. The Nation, 4.3.2000; 16.2.2000; Bangkok Post, 5.3.2000.

42

Generalleutnant der Polizei Kowit Watana, Assistant Commissioner zur Untersuchung von Wahlverstößen bei der Wahlkommission (zitiert in Bangkok Post vom 2.3.2000). 
Stimmenkauf verwendet wurde, ist unklar. Als nützlicher Indikator für die Überprüfung ob und in welchem Umfang Stimmenkauf praktiziert wurde, erwies sich in der Vergangenheit der Blick auf Veränderungen in der Bargeldzirkulation während der Wahlperiode. ${ }^{43}$ In den besonders von diesen Praktiken betroffenen Regionen des Landes ließ sich bei Wahlen in der Vergangenheit eine deutliche Zunahme im Geldumlauf bei Banknoten mit kleinem Nennwert feststellen, jenem Zahlungsmittel, daß sich zum Stimmenkauf besonders eignet. Dieses insbesondere für die ärmeren Regionen des Landes typische Phänomen war auch diesmal festzustellen. So wurden nach Angaben der thailändischen Zentralbank in der nordöstlichen Region des Landes im Februar 2000, einen Monat vor den Wahlen, 41,8 Prozent mehr kleine Geldscheine (10, 20 und 50 Baht) ausgezahlt als im gleichen Monat des Vorjahres; im Süden waren es 27,5 Prozent und im Norden 11,1 Prozent (zit. nach Bangkok Post 4.3.2000). In der südlichen Provinz Yala verringerte sich in den drei Tagen vor der Wahl die Zahl der bei Geschäftsbanken verfügbaren Geldscheine mit kleinem Nennwert um 70 bis 80 Prozent. ${ }^{44}$

Stimmenkauf und der Verkauf der eigenen Stimme stellen für sich betrachtet keine Einschränkung der Bedeutsamkeit demokratischer Wahlen dar: die freie Verfügung des Einzelnen über sein Wahlrecht und damit verbunden die Freiheit, das eigene Wahlrecht wahrzunehmen oder nicht wahrzunehmen beinhaltet logischerweise auch die freie Wahl der Entscheidungsgründe. Wähler sind in der Regel nicht materiell gezwungen, ihre Stimme zu verkaufen $^{45}$; ob ein Wähler tatsächlich seinen Teil des Abkommens erfüllt, kann vom Käufer der Stimme im Einzelfall nicht überprüft und ex post nicht sanktioniert werden. Die direkte Zahlung von Geldbeträgen stellt zudem nur eine von mehreren Formen der "monetären" Beeinflussung von Wahlentscheidungen dar. Weitere und im thailändischen Kontext besonders bedeutsame Formen wie Spenden an öffentliche oder religiöse Einrichtungen im Wahlkreis oder das Versprechen auf zukünftige Zuwendungen an den Wahlkreis durch den gewählten Abgeordneten (pork barrels) werden hierdurch ebenso nicht erfaßt, wie das Versprechen, nach der erfolgreichen Kandidatur eine "Dividende" an die Wähler auszuschütten. ${ }^{46}$ Gleichwohl ist klar, daß Stimmenkauf als politisches Syndrom die demokratie-

So weisen im thailändischen Kontext zwei Merkmale daraufhin, daß Stimmenkauf nur bedingt auf materielle Not der Wähler zurückgeführt werden kann: Erstens sind die gezahlten Beträge in der Regel zu gering, um einen ausreichenden finanziellen Anreiz zu bieten; zweitens findet Stimmenkauf in allen Regionen des Landes statt, auch in den wohlhabenden Gebieten der Hauptstadt und der umliegenden Zentralregion sowie im Süden des Landes. Ich danke Uwe Solinger für diesen Hinweis. tischer Korruption bei Wahlen vgl. Anusorn Limmanee, ibid.; Anek Laothamatas, A Tale of Two 
theoretische Bedeutung des aktiven Wahlrechts ad absurdum führt. Das dieses Problem auch in Thailand selbst als solches erkannt wird, zeigen die intensive öffentliche Diskussion zu diesem Thema, das gesetzliche Verbot von Stimmenkauf (mit zumindest hypothetisch erheblichen Sanktionsmöglichkeiten), die explizit mit dem Ziel der Eindämmung von Stimmenkauf begründete Änderung des Wahlsystems für das Repräsentantenhaus sowie die Schaffung der EC. Gleichwohl zeigte die Senatswahl, daß die Skepsis von Beobachter, wie auch Mitglieder der EC selbst hinsichtlich der Möglichkeiten zur Durchsetzung dieser Regeln ${ }^{47}$ angebracht war.

\section{Bewertung der Senatswahlen}

Die Bewertung der Wahl muß ambivalent ausfallen. Zum einen markierte sie einen Fortschritt in der demokratischen Entwicklung Thailands, da hiermit die letzte institutionelle "reserved domain" der Militärs und Bürokraten demokratisch geöffnet wurde. Von Seiten der thailändischen Presse und zivilgesellschaftlichen Gruppen wurde der Ausgang der Wahlen überwiegend als Schritt zur Festigung der Demokratie begrüßt. Befürchtungen bezüglich einer "Unterwanderung" des Senats durch parteipolitisch gebundene Kandidaten zum Trotz war die politische Zielsetzung zumindest in Bangkok erreicht worden. Bei den meisten Gewählten handelte es sich tatsächlich um Personen, die für ihr sozialpolitisches Engagement und ihre Integrität bekannt waren. ${ }^{48}$ Der Anteil dieser Kandidaten an den im ersten Wahlgang gewählten Senatoren betrug jedoch insgesamt nur etwa drei Prozent aller Senatoren. Ebenso wie der Frauenanteil im neuen Senat $(9,5 \%)$ lag er damit letztlich erheblich unter den Erwartungen. Vielmehr gehörten 38\% aller neuen Senatoren "politischen Familien" (Politiker und deren Verwandte) an, bei weiteren 34\% handelte es sich um pensionierte Beamte und knapp 15\% der Gewählten waren Unternehmer. Schätzungsweise rund $40 \%$ der neuen SenatorInnen besaßen ungeachtet des Verbots formaler Mitgliedschaft in einer Partei direkte parteipolitische Verbindungen. Die Wiederholung der Wahl in einigen Provinzen veränderte dieses Bild nur bedingt. Von den insgesamt 78 annullierten Siegern gewannen 45 auch im zweiten Wahlgang (Bangkok Post 30.4.2000). Verstöße gegen das Wahlgesetz wurden in 30 der 35 Provinzen gemeldet. Erneut wurden die restrik-

Democracies: Conflicting Perceptions of Elections and Democracy in Thailand, in: Taylor, $R$. $H$. (ed.), The Politics of Elections in Southeast Asia, 1996, S. 201-223; Robertson, Philip S., The Rise of the Rural Network Politician: Will Thailand's New Elite Endure, in: Asian Survey 36 (1996), Nr. 9, S. 924-941; Callahan, William A. / McCargo, Duncan M., Vote-Buying in Thailand's Northeast. The July 1995 General Elections, in: Asian Survey 36 (1996), Nr. 4, S. 376-392.

Suchitra, Punyaratabandhu, Thailand in 1997. Financial Crisis and Constitutional Reform, in: Asian Survey 35 (1998), vol. 2, S. 161-167: 165; Ockney, James, Thailand: The Crafting of

Democracy, in: Southeast Asian Affairs 1998, S. 301-316.

Bangkok Post, 9. März 2000 und 12. März 2000. 
tiven Bestimmungen zum Wahlkampf und das Verbot von Stimmenkauf vielfach mißachtet, so daß die Wahlbehörde sich entschloß, in neun Provinzen für insgesamt zwölf Mandate sogar einen dritten Wahlgang (Juni 2000) anzusetzen. ${ }^{49}$

Die Tatsache, daß erstmals in der thailändischen Wahlgeschichte die gesetzlichen Bestimmungen tatsächlich angewendet, Ergebnisse aufgrund von Unregelmäßigkeiten aufgehoben, Kandidaten wegen vermuteter Verstöße gegen das Wahlgesetz vor Gericht gestellt und verurteilt wurden und die für die Durchführung der Wahlen zuständigen Organe sich relativ unabhängig gegenüber politischen Druck von Seiten der Regierung und des politischen Establishments erwiesen, ist grundsätzlich positiv zu bewerten, weist aber auch einige problematische Aspekte auf. Deutlich wurde in beiden Wahlgängen, daß das thailändische Wahlgesetz zahlreiche Bestimmungen aufweist, die schlicht nicht umsetzbar sind, oder in etablierten westlichen Demokratien wohl kaum zu Beanstandungen der Wahl geführt hätten. Während das Wahlkampfverbot zumindest in Bangkok weitgehend im Sinne des Gesetzgebers wirkte und gesellschaftspolitisch engagierte Kandidaten ihren Bekanntheitsgrad erfolgreich in Stimmen umsetzen konnten, zeigte das Verbot in den ländlichen Gebieten, die ca. 80\% der Wählerschaft stellen, die umgekehrte Wirkung. Während die bestehenden electoral machines der Parteien und Politiker von der Wahlbehörde nur begrenzt behindert werden konnten, entzog das Verbot unbekannten, "neuen" Gesichtern die Möglichkeit, ihren Bekanntheitsgrad unter der Wählerschaft zu steigern. Die Vertreter der etablierten politischen Klasse kamen somit entgegen der Intention des Gesetzgebers vermutlich zu wirkungsvollen Wettbewerbsvorteilen, die sich besonders deutlich in der hohen Zahl gewählter Ex-Parlamentarier und Verwandten oder Freunden amtierender Minister und Abgeordneter niederschlugen.

Was die korrekte Durchführung der Wahlen betrifft, kann die Senatswahl als vorsichtiger Schritt hinzu mehr "election quality" ${ }^{50}$ gelten, insbesondere aufgrund der entschlossenen Haltung der Wahlkommission. Obwohl sich die Wahlbehörde mit ihrer Entscheidung zur Annullierung der Wahlen in 35 Provinzen standhaft gegenüber Versuchen der politischen Beeinflussung zeigte, war diese Maßnahme aber keineswegs unproblematisch. So erfolgte die teilweise Suspendierung der Ergebnisse auf einer schwachen rechtsstaatlichen Grundlage: Lediglich in fünf Fällen lagen der EC ausreichende Beweise vor, um die Sieger vom zweiten Wahlgang auszuschließen. Die übrigen 73 Kandidaten mußten erneut zugelassen werden, da ihnen kein persönliches Fehlverhalten nachzuweisen war. ${ }^{51}$ Es stellt sich zudem die Frage, nach welchen Kriterien die Wahlbehörde entscheiden konnte, ob ein Teil der

50 Elklit, Jorgen, Electoral Institutional Change and Democratization: You Can Lead a Horse to Water, But You Can't Make it Drink, in: Democratization 6 (1999), vol. 4, S. 28-51: 34. 
Wahlergebnisse in einem Distrikt nicht korrekt, ein anderer Teil im selben Distrikt jedoch gültig war. Selbst wenn angenommen wird, daß die beanstandeten Ergebnisse aufgrund von Verstößen gegen das Wahlgesetz zustande gekommen waren, stellt sich die Frage, anhand welcher Kriterien die Kommission innerhalb eines Wahlkreises zwischen gültigen und ungültigen Ergebnissen differenzieren konnte. Die konsequente Lösung wäre sicherlich gewesen, bei Verstößen gegen das Wahlgesetz das gesamte Ergebnis in einem Wahlkreis zu annullieren.

\section{Ausblick: Die Bedeutung der Wahl für die zukünftige Entwicklung der Demokratie}

Die Bedeutung der Wahl für die weitere Konsolidierung der Demokratie in Thailand ist unter einem doppelten Blickwinkel zu betrachten: zum einen mit Blick auf die zukünftige Entwicklung der politischen Ökonomie von Wahlen sowie zum anderen mit Blick auf die Rolle des neu gewählten Senats. Hinsichtlich des zuerst genannten Aspekts läßt sich feststellen, daß die Wahl nur teilweise die Hoffnung auf einen Bruch mit den bisherigen Praktiken von "electoral politics" erfüllt hat. Die Begleitumstände der Wahl haben verdeutlicht, daß hinsichtlich der Institutionalisierung "bedeutsamer" Wahlen (Hadenius 1992) noch erhebliche Defizite bestehen; dies gilt vor allem für die korrekte Durchführung der Wahlen. Die durch wahltaktische Überlegungen zahlreicher Kandidaten motivierten Verstöße gegen das Wahlgesetz haben erneut gezeigt, daß die attitudinale Verpflichtung der politischen Elite des Landes gegenüber den zentralen demokratischen Prinzipien schwach ist. Obwohl ein Trend zur Verbesserung der qualitativen Standards bei dieser Wahl unverkennbar war, haben sich die alten Mechanismen und Taktiken zur Akkumulation von Wählerstimmen durch Kandidaten erneut als wirksam und beharrungsfähig erwiesen.

Aus konsolidierungs- und demokratietheoretischer Perspektive bedenklich erscheinen zwei weitere Merkmale der jüngsten Wahl. Erstens ist die unter rechtsstaatlichen Gesichtspunkten problematische Entscheidung der Wahlbehörde zu nennen, ohne ausreichende Beweislast gegenüber den betroffenen Kandidaten Wahlsiege zu annullieren. Sie demonstriert einerseits die Unabhängigkeit der Kommission, zumal sich unter diesen Bewerbern zahlreiche Verwandte und Freunde von Regierungsmitgliedern und hochrangigen Politikern

befanden. ${ }^{52}$ Andererseits hat die Wahlbehörde mit dieser, wie Äußerungen ihrer Mitglieder den Schluß nahe legen, politisch motivierten Entscheidung jedoch, ihre politische Neutralität aufgegeben und den gezielten Versuch unternommen "unerwünschte" Kandidaten aus dem Senat fernzuhalten. Auch wenn diese Vorgehensweise auf Zustimmung der Medien und zivilgesellschaftlicher Organisationen stieß, bleibt die Erkenntnis, daß die Wahlkom- 
mission damit einen Präzedenzfall geschaffen hat, auf den sich einer weniger "reformorientierte" Kommission mit gegenteiliger Intention in Zukunft stützen könnte.

Die rigide Auslegung des Wahlgesetzes durch die Wahlkommission hat zudem bewirkt, daß die zweite Kammer bis zum Juni diesen Jahres nicht zu ihrer konstituierenden Sitzung zusammentrat. Wie das Verfassungsgericht in seiner Entscheidung vom April diesen Jahres feststellte, kann die zweite Kammer erst dann ihre Arbeit aufnehmen, wenn alle 200 Senatoren gewählt und von der Wahlkommission bestätigt wurden. ${ }^{53}$ Diese Interpretation des Wahlgesetzes ist für die für die zweite Jahreshälfte bevorstehenden Wahlen zum Repräsentantenhaus von eminenter Bedeutung. Sollte die Wahlkommission in ähnlicher Weise vorgehen, so droht die Handlungsunfähigkeit des gesamten politischen Systems, da in diesem Fall auch das Repräsentantenhaus nicht zusammentreten könnte, womit sich die Wahl des Premierministers erheblich verzögern und das gesamte politische System lahmgelegt werden könnte. Wenig erstaunlich ist daher, daß nach dem zweiten Wahlgang vom 29. April erheblicher Druck auf die Wahlkommission ausgeübt wurde, fragliche Sieger zunächst anzuerkennen und erst nach dem Zusammentreten des gegen diese vorzugehen.

Zweitens ist der gezielte Ausschluß politischer Parteien zu nennen. Der häufig beklagte Umstand, daß es den thailändischen Parteien an gesellschaftlicher Verankerung mangele, ihre Responsivität gegenüber gesellschaftlichen Forderungen und ihre Fähigkeit zur Artikulation sozialer Interessen gering sei und Versuche der Institutionalisierung eines repräsentativen, sozial und politisch inklusiven sowie programmatisch ausdifferenzierten Parteiensystems bislang allesamt gescheitert seien, ist zutreffend. ${ }^{54}$ Thailändische Parteien ähneln eher den europäischen Elitenparteien des 19. Jahrhunderts, als den Kartellparteien des ausgehenden 20. Jahrhunderts, von den Massen- oder Volksparteien der west- und mitteleuropäischen Demokratien des 20. Jahrhunderts nicht zu reden. Der Versuch, eine zweite, meritokratische Kammer zur Kontrolle der ersten Kammer zu schaffen, kann in einer solchen Situation eine mögliche Strategie zur Stärkung der demokratischen Qualität politischer Repräsentation sein. Allerdings hat die Wahl gezeigt, daß der Versuch, eine solche Kammer durch Wahlen zu besetzen, den politischen Gegebenheiten der thailändischen Gesellschaft nicht gerecht wird. In einem politischen Umfeld, in dem Parteien, so gering ihr Beitrag zur demokratischen Partizipation der Bürger im konkreten Fall auch sein mag, als "Wahlmaschinen" bereits etabliert sind, verfügen sie bzw. die von ihnen unterstützte Kan-

Vgl. Croissant, Aurel, Genese, Funktion und Gestalt von Parteiensystemen in jungen asiatischen Demokratien, in: Merkel, Wolfgang / Sandschneider, Eberhard (Hrsg.), Systemwechsel 3. Parteien im Transformationsprozeß, Opladen, 1997, S. 293-337; Anusorn (Fn. 10); Dosch, Jörn, Der beschwerliche Weg zur rechtsstaatlichen Demokratie in Südostasien, in: Aus Politik und Zeitgeschichte 2000, B 21, S. 16-23. 
didaten gegenüber parteilich nicht gebundenen Bewerbern über ganz erhebliche organisatorische Wettbewerbsvorteile, die es unwahrscheinlich erscheinen lassen, daß die Mehrzahl der Kandidaten als rationale "vote seekers" auf die Nutzung solcher Vorteile verzichten. Die Umsetzung des meritokratischen Prinzips im Rahmen allgemeiner, kompetitiver Wahlen erscheint aus dieser Perspektive kaum möglich. Zudem stellt die öffentliche Diskussion um die Einführung eines "anti-parteilichen" Senats einen klaren Rückschritt in die Argumentationsmuster der 70er und 80er Jahre dar, als Parteipolitik mit Korruption gleichgesetzt wurde. Der Ausschluß von Parteien vom politischen Wettbewerb vermag kaum zu ihrer Stärkung und Demokratisierung beizutragen.

Welche Rolle schließlich der neu gewählte Senat zukünftig in der thailändischen Politik spielen wird, ob es dem Senat gelingen wird, sich von eigenen institutionellen Traditionen zu lösen und die Rolle einer "nationalen", politikgestaltende Kammer zu besetzen, als Kontrollorgan der Exekutive und seines Gegenparts in der Legislative ein scharfes Profil zu entwickeln und ob die zweite Kammer den deliberativen Charakter der politischen Auseinandersetzung stärken kann, wird in besonderem Maße davon abhängen, ob die neuen den Senatoren den schwierigen Spagat zwischen Kontrolle und Kooperation bewältigen werden. Die konstitutionellen und politischen Ausgangsbedingungen hierfür sind gemischt. Einerseits verfügt der Senat nach der Wahl sowohl über die verfassungsrechtlichen Kompetenzen, als auch über die plebiszitär-demokratische Legitimität, um Regierung und Repräsentantenhaus gegenüber selbstbewußt aufzutreten. Andererseits ist fraglich, ob die 200 neuen Senatoren den hierfür notwendigen politischen Willen aufbringen werden, ob die zweite Kammer die vorprogrammierten Probleme beim Aufbau eines effizienten intraparlamentarischen Verhandlungssystems bewältigen kann und ob Regierung und Repräsentantenhaus bereit sein werden, den Senat als institutionellen Verhandlungspartner zu akzeptieren. Andernfalls droht der Senat und mit ihm das gesamte Regierungssystem im Spannungsfeld zwischen deliberativer Politik und politischer Blockade gefangen zu bleiben. 